\title{
Design and Discussion of Multimedia Interaction Technology based on Virtual Reality
}

\author{
Yu Fan ${ }^{1, \text { a }}$, Jia Liü, b \\ ${ }^{1}$ Haikou College of Economics, Haikou, Hainan, 571100 \\ ${ }^{a}$ email, ${ }^{b}$ email
}

Keywords: Virtual reality; Multimedia interaction; Interaction

\begin{abstract}
With the continuous development of society, people have more and more material and spiritual needs. The interactive design of multimedia has drawn people's attention. Virtual reality can make people feel the immersive experience. Nowadays, many virtual museums and virtualized operating platforms have appeared in the people's real life. The application of these platforms has adopted a large number of multimedia technologies and has broad prospects for social development. Its application scope is constantly perfect. The development of multimedia interactive design is also more and more sense of direction, giving people more imagination.
\end{abstract}

\section{Introduction}

Human beings in the process of receiving information not only through the visual, while hearing, touch, smell and taste have played a very important role, but 95\% of the information is obtained through visual, auditory and tactile. Humans in dealing with the information, the use of multi-sensory approach to handling, very convenient. Nowadays, although computer technology has brought a lot of conveniences to people, it still can not reach the natural level of human beings. In the process of information exchange, computer is still a single source of information. Humans have had very close connections with computers, and people can access vast amounts of information effortlessly, but much of it is reused. Multimedia refers to a mixture of sound, image and data. Multimedia technology gives a very complex feeling, but it is just a mixture of multiple perception technologies. In the process of perceiving things from the outside world, people are limited in their ability to perceive and contact with the entity. Although visual displays have made great strides, tactile senses are mostly keyboards and mice in most cases. Hearing is also an important part of the interaction, but the current changes are not particularly obvious. Smell and taste have not been well developed yet. For the development of multimedia, the development of multimedia interactive design of virtual reality also has a very bright future.

\section{The concept and main features of virtual reality}

In recent years, with the continuous development of social economy, the development of multimedia interactive technology has been extensively used in multimedia design and its development has been rapidly changing. In the use of virtual reality technology, technicians can use the computer three-dimensional technology to simulate the environment and space around them, and make people have the immersive feeling through the interaction design.

Virtual reality technology was developed in the eighties of the last century. It is a type of perceptual simulation system whose main purpose is to improve the human-computer interaction so that the user can have a kind of three- Immersive experience, the audience seems to be among them. The audience can fully adopt the virtual reality technology, which can simulate the real world and respond to the user's action. Users and the virtual environment can establish an interactive relationship, bring people a realistic feeling.

There are three main characteristics of virtual reality technology, one is interactive, the other is immersion, and the third is conception. Interactivity mainly refers to the user can manipulate the actual object in the virtual environment, and then the specific environment can be fed back. The way of this interaction mainly adopts the way of three-dimensional interactive device, and people 
can use the data glove and the sensing device the way. Immersion refers to people in the virtual environment, you can feel the real environment exists, with full use of their own visual perception, auditory sense and tactile perception, you can get the actual experience. Perfect virtual reality technology should include five kinds of human perception, conception refers to people in many unknown areas can get some sensibility and rationality, these ideas can be fully applied to the field of medical and aerospace.

\section{Virtual reality technology in the design of multimedia interaction and the development trend}

In a relatively complex multimedia system, interactive design should be based on the user's habits, in the construction of virtual reality environment, if the user is very familiar with the application, then their learning costs will be reduced, users get what they want The result will be more easy. Users can obtain more psychological recognition in specific experience, which can reduce user's wrong operation in use, reduce the sense of loss caused by the artwork to the user, and make the interaction become a more pleasant experience.

For example, in the multimedia project, "Adults Love Science," this work was designed by students who used multimedia to build an assembly model in which people could use a mouse to control a virtual laboratory. People's curiosity Can be fully satisfied, in this new environment, people can get more experience.

After users have a general idea of the new environment, they can find out what they are interested in and deepen the interaction. In the traditional interface, the general use of the flat interface approach, in the interface switching, the general is to achieve through navigation. However, the navigation bar is rendered by means of text, and even if there is an image, it is a simple graphic. In "Lover Love Science", a three-dimensional virtual laboratory is built by means of a three-dimensional model. The entire interface is three-dimensional. The user can use his own sense of direction to determine the position of the interface. In the first perception, the user Impressions may not be deep enough, but they have a preliminary understanding of the location of the interface. In this work, you can respect people's habits and use the right-hand mouse operation.

In the multimedia work "Listening to Voices," a large number of virtual reality techniques are used. In this work, people are born into the real scene of Danzhou in Northern Song Dynasty 900 years ago. In the process of designing works, the commentary is used by the first person, the screen is the use of full-screen mode, the screen using shaft-type presentation, people seem to walk in the $528 \mathrm{~cm}$ "Qingming Riverside" in the picture Unfolding in the process, people can understand every detail in the picture, bring people beautiful scenery.

In order to make the readers more clearly understand the bustling scenery of the time, the works used the virtual reality technology to reappear the beauty of 900 years ago in front of the audience. The original appearance of the transportation and architecture at that time reflected the fact that at that time Social characteristics and cultural characteristics.

In real life, we have learned a great deal of content from museums and science and technology museums about history and science and technology. They can show ancient history and social scenes. Relevant statistical analysis shows that there are nearly 400,000 cultural relics sites registered in China, with a total of 12 million cultural relics and a large number of new cultural relics unearthed each year. However, because of the limited time, the protection of cultural relics is subject to many restrictions, and a very small number of cultural relics can be exhibited. However, in the application of virtual reality technology, the display of cultural relics can break the boundaries of space and time. Through the network technology, people can truly feel the atmosphere of the museum. The extension of the museum space will also be greatly improved. The function of the museum can also be extensive The show, to meet the general needs of the public. In the construction of the virtual museum, with the aid of digital simulation technology, the real three-dimensional model scene can be analyzed and the scenario can be interactively reproduced. In the process of virtual item display, a large number of scenes can be stored in the form of data in the computer. As the archeology technology continues to improve, people can get more information, the scene virtual way, you can make the scene truly reproduce. 
In the Shanghai World Expo, the use of virtual reality technology, venues for the Expo panoramic view of the reproduction of the scene to restore the true. Visitors can easily visit the World Expo with computers and the Internet. In the virtual space, you can easily view the details of Expo with a multi-angle approach. Now the Shanghai World Expo online exhibition hall has exceeded 300 million hits.

Virtual reality technology can be used in urban planning, play a good publicity in the construction of the city, to enhance the image of the city. And the system will analyze the status quo of the city and then make plans for the future. Design the city's past, present and future.

Virtual reality technology can also be widely used in the construction of online museums and science and technology museums. Many digital science and technology museums and digital planning museums in China have adopted virtual interactive multimedia technology to achieve a high degree of digital restoration.

Virtual reality technology can be applied to the virtual reproduction network of physical products for sale. A large number of virtual stores have appeared on the network. These stores adopt a high degree of fidelity technology and can simulate real store scenes. Even if people stay at home , Can also buy products. In the online $4 \mathrm{~S}$ shop, people can build 3D data model, through the computer terminal, customers can observe the new look and design in all directions, but also simulate the real scene, people can enter the car, feel the car Decoration, feel the space inside the car. Can also simulate driving, feeling like playing a game, the car into the road.

Virtual reality technology has also been widely used in education, which is mainly manifested in the hands-on experience. Students can perceive the truth through various types of simulation experiments.

In virtual demonstration, virtual reality technology has been widely used in the parade on the simulation software can be used to simulate the rehearsal, the drill, the staff can determine the speed of the square matrix can also predict the parade Effect.

In the study of virtual reality technology, there is still a long way to go, but we should also give full play to their imagination so that the virtual reality technology to achieve a breakthrough.

The Application of Virtual Reality Technology of Somatosensory Technology in Multimedia Interaction Design

Nowadays, multimedia electronic devices are widely used in all fields of society. The devices are very diverse and can be used by mobile phones, mobile TVs and computer devices. So in the near future there will be more media, these media to entertainment and messaging as the main purpose, people's multiple senses will get more experience in virtual reality technology. The technology that uses different senses to interact in virtual reality technology is called virtual interactive multimedia system.

As early as 2006, Japan introduced a video game that gives people an immersive gaming experience in which people wave their racquets as if their arms were stretching. We do aiming at the game action, so in reality, to use the remote control.

The United States also launched a virtual reality-based game in 2010, in which gameplay has been changed. In the game, people no longer need to use the remote control, and people can really become the protagonist of the game. The game's operation is very simple, the use of body movements can replace the remote control, which will be based on people's actions to make a specific response, when people move or jump in the link, the sensor will be promptly processed Various actions, the role of the game will also play a direct move and jump action. When the player plays the game for the second time, the software can be identified directly, then simply add the game. In the game, can not shake the limbs, but also can use facial expressions.

In the market, there are many models of mobile phones using the induction system, mobile phone induction system refers to the people in the use of mobile phones, you can flip the screen like a book, to achieve the page slide and find ways. And all kinds of induction games installed on the phone, these games, people can be issued by the action, the direct control of the game's hero.

Now, there are many applications in mobile phones with gravity sensing. Body full of mobile phone systems with virtual reality technology, but also with the help of interactive projection. 
Interactive projection is a type of capture and capture technology based on many infrared sensing contacts in a real-world space. With the help of image analysis system, the captured motion can be found. This data can be Combine real-time images to create direct interaction between the viewer and the screen, which is fully utilized in many large exhibitions, design the relevant scene in the showroom, and then viewers walk into the scene, The audience can feel the wind blowing, but also feel the feeling into the grass.

In commercial applications, the combination of somatosensory technology and virtual reality technology can be utilized in many commercials in public places to attract a large audience. In the projection image area layout of the link, should be combined with the appropriate size to ensure that the size of the projection and the surrounding environment is better, according to different interactive content, improve the entertainment effect. In the demonstration session, the audience is no longer out of the woods, but embedded into the specific environment, you can experience in person. This novel way of experience empowers passers-by to resonate with advertisements, increasing the desire to reach the product so that the protagonist can better integrate into the corresponding scenarios, leaving long-term memories and improving the publicity of the product.

Nowadays, people have entered the digital era, in which people have come into contact with the digital development has been achieved. Whether young or old, whether in school or in the workplace, multimedia systems have gained a very wide range of applications that enrich people's lives and are an important part of the entertainment and learning culture of people.

Virtual reality multimedia interaction technology can be used in different people, giving people a strong visual impact to meet people's needs in all directions, but also spawned a lot of new ideas, more than simple virtual interactive multimedia technology Practical, combined with visual, auditory and tactile, so that the virtual reality interactive multimedia become more diversified.

\section{Conclusion}

Virtual reality technology has a high degree of presence, the only part of the simulation of a specific environment, you can feel the real world. And it achieves a friendly, interactive feature that can be manipulated directly in the environment and fed back in the simulated environment. Used in the crawling of the environment in the part of the link, you can feel the weight of the object. Multi-perception of virtual reality technology is very prominent, in addition to the general computer's ability to perceive, it also has auditory perception, tactile perception. In VR technology, users can achieve autonomy in which objects behave according to the laws of physics. Virtual Reality combines computer technology to create a realistic visual, auditory and tactile virtual environment. With the help of the necessary equipment, users can manipulate the objects in the virtual environment to obtain a realistic experience. With virtual reality technology, improve the analysis of virtual entities, combined with the user's real physiological experience, in the simulated environment to achieve interaction. Through systematic analysis, the virtual reality technology mainly includes the detection module, the feedback module and the sensor module, and these modules can all be generated in the form of modeling. In the aspect of the application of dynamic modeling technology, three-dimensional data of the real environment can be generated, and a corresponding virtual illusion model can be established.

\section{Acknowledgements}

Project Title: (School level) "Smart Community" smart product design and research (project number: hjky17-10)

Project Name: Research and Practice of Establishing South Lost Road Cultural Display Platform Based on "Internet Plus" Mode (Project Number: Hnky2017-63)

\section{References}

[1] Chen Jun, Cai Jinling. Design and Fabrication of 3D Multimedia Courseware Based on Unity3D 
[J] .Modern Computer, 2014, (34): 64-67.

[2] Zhang Zhian, Lei Peiying, Wang Yue. Study on Campus Guidance Identification System Based on Virtual Reality Technology [J]. Journal of Xi'an University of Arts and Science (Natural Science Edition), 2011,14 (03): 99-102.

[3] Su Zhiyong. Multimedia Courseware Development Based on Virtual Reality Technology [J] .Journal of Science and Education (Mid V Journal), 2011, (06): 27-28. 